\title{
Effect of Scutellaria Baicalensis Georgi Stems and Leaves Flavonoids on Multisite Phosphorylation of tau Protein Induced by Composited A $\beta$ in Rats
}

\author{
Liu Qianqian, Ding Shengkai, Shang Yazhen* \\ Institute of Traditional Chinese Medicine, Chengde Medical College / Hebei Province Key Research Office of \\ Traditional Chinese Medicine against Dementia / Hebei Province Key Laboratory of Traditional Chinese \\ Medicine Research and Development, An Yuan Road, Chengde 067000, PR China.
}

*Corresponding Author: Shang Yazhen, Institute of Traditional Chinese Medicine, Chengde Medical College / Hebei Province Key Research Office of Traditional Chinese Medicine against Dementia / Hebei Province Key Laboratory of Traditional Chinese Medicine Research and Development, An Yuan Road, Chengde 067000, PR China.

\section{Abstract:}

\section{Objective}

To investigate the effect of Scutellaria Baicalensis Georgi stems and leaves flavonoids (SSF) on multisite phosphorylation of tau protein in hippocampus and cerebral cortex induced by amyloid beta 25-35 (A $\beta_{25-35)}$ combined with aluminum trichloride $\left(\mathrm{AlCl}_{3}\right)$ and recombinant human transforming growth factor- $\beta 1$ (RHTGFB1) (composited $A \beta$ ) in rats.

\section{Methods}

Sixty healthy male Wistar rats were intracerebroventricular microinjected $A \beta_{25-35}$ combined with $A l C l_{3}$ and RHTGF- $\beta 1$ to establish the model of mimic Alzheimer's disease (AD). After 45 ds of operation, screening of successful memory impairment models were performed with Morris water maze. The successful memory impairment models randomly divided into model group and three doses of drug groups. The rat in the drug group intragastrically administered 35, 70 and $140 \mathrm{mg} / \mathrm{kg} S S F$ for $30 \mathrm{ds}$. The protein expression levels of p-tau (Thr205), p-tau (Thr212), p-tau (Ser202), p-tau (Ser205), p-tau (Ser214) and p-tau (Ser356) in hippocampus and cerebral cortex of rats were detected by Western blotting method.

\section{Results}

Western blotting results showed that the expression of p-tau (Thr205) and p-tau (Ser214) protein were significantly increased, p-tau (Ser202), p-tau (Ser205) and p-tau (Ser356) were decreased and p-tau (Thr212) was no significant difference of the model group in the hippocampus and cerebral cortex, as compared with the sham group. The rat were intragastrically administered for $30 \mathrm{ds}$ with 35, 70, and $140 \mathrm{mg} / \mathrm{kg} \mathrm{SSF}$, compared with the model group, the expression of p-tau (Ser214) protein was reduced, p-tau (Ser356) protein was increased, and p-tau (Thr205) and p-tau (Ser202) were not significantly change in the hippocampus. The expression of p-tau (Thr205), p-tau (Ser202), p-tau (Ser205), p-tau (Ser214) and p-tau (Ser356) were lessened, p-tau (Thr212) showed no significant change in the cerebral cortex of rats.

\section{Conclusion}

Intracerebroventricular microinjection of $A \beta_{25-35}$ combined with $A l C l_{3}$ and $R H T G F-1$ can induce multiple tau protein phosphorylation in the brain. SSF can modulate tau phosphorylation, especially inhibit the tau phosphorylation at Ser205 and Ser214 sites, which suggests that improvements of SSF in neuroprotection and memory disorders may be related to its regulation in multiple sites hyperphosphorylation of tau.

Keywords: Scutellaria Baicalensis Georgi stems and leaves flavonoids; tau hyperphosphorylation; p-tau (Thr205); p-tau (Ser214); Alzheimer's disease;

\section{INTRODUCTION}

Alzheimer's disease $(\mathrm{AD})$ is a neurodegenerative disease dominated by progressive memory loss. Its hallmark pathological feature are abnormal deposition of senile plaques from amyloid $\beta$-protein $(\mathrm{A} \beta)$ and neurofibrillary tangles (NFTs) of proteinpaired helical filamend (PHF) from perphosphorylated tau protein ${ }^{[1]}$. Abnormally hyperphosphorylated tau protein can cause NFTs to lose normal biological functions, resulting in neuronal toxicity, damaging the normal structure of microtubule-associated 
proteins, and then inducing neuronal degenerative diseases. It is reported that the severity of AD patients is positively correlated with the density of NFTs ${ }^{[2,3]}$. Tau phosphorylation, the most critical factor in the pathogenesis of $\mathrm{AD}^{[4]}$, is an important biological marker for evaluating the degree of disease for $\mathrm{AD}$ patients. Tau phosphorylation mainly occurs on serine or threonine residues, its phosphorylation level is regulated by tau protein kinase, which are principally included cyclin deendent kinase-5 (CDK-

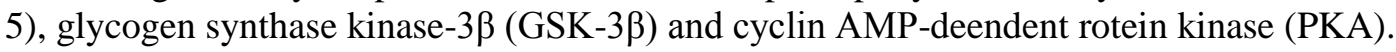

Scutellaria Baicalensis Georgi (SSF), a flavonoid, isolated from aerial parts of radix Scutellariae Labiatae plants, has been confirmed that the effect of antibacterial, antioxidant, as well as improvement memory impairment and promote nerve regeneration ${ }^{[5-7]}$. The effects of SSF on the phosphorylation level of tau protein at multiple sites induced by composited $A \beta$ in hippocampus and cerebral cortex of rats has not been reported. Here, in the present study, intracerebroventricular microinjection of composited $\mathrm{A} \beta$ to establish a model of mimic AD memory impairment, the effects of SSF on multisite phosphorylation of Thr205, Thr212, Ser202, Ser205, Ser214 and Ser356 of tau protein in the hippocampus and cerebral cortex were reported.

\section{MATERIALS AND METHODS}

\subsection{Animals, Instruments and Reagents}

Healthy adult male Wistar rats were purchased from Beijing Vital River Laboratory Animal Technology Co., Ltd (SPF grade, Certification No. SCXK (Ji) 2016-0006). Rats were housed in groups of five per cage with free access to food and water, under controlled laboratory conditions for 12-hours light-dark cycle and ambient temperature of $23 \pm 1^{\circ} \mathrm{C}$. The rats were allowed to acclimatize to the laboratory environment for a week before the operation. All the experimental animals received humanistic care, and their use was approved by the Animal Ethics Committee of Chengde Medical College under the serial number CDMULAC-20190226-002. In addition, all efforts were made to minimize number of animals used and their discomfort. Brain Stereotactic Instrument was purchased from Shenzhen Rayward Life Technology Co., LTD. Morris water maze was supplied by Institute of Materia Medica, Chinese Academy of Medical Sciences. Electrophoresis System was purchased by Bio-Rad Company. IMAGER550 Gel Image Analysis System was provided by ALPHA Corporation, USA).

SSF, purity is $86.8 \%$, provided by The Institute of Chinese Medicine at Chengde Medical College. $\mathrm{A} \beta_{25-}$ 35 (Lot. MB10445) was purchased from Dalian Meilun Biotechnology Co., Ltd. $\mathrm{AlCl}_{3}$ was provided by Tianjin Beichen Chemical Reagent Company, and RHTGF-1 was purchased from PEPROTECH Company. Antibodies p-tau (Thr205) (Lot. AB181206), p-tau (Thr212) (Lot. AB4842), p-tau (Ser202) (Lot. AB 108387), p-tau (Ser205) (Lot. AB201784), p-tau (Ser214) (Lot. AB170892) and p-tau (Ser356) (Lot. AB92682) were purchased from Abcam campany. Reference $\beta$-actin (Lot. YM3028) was purchased from Immunoway, Co., Ltd. and sheep Anti-rabbit IgG (Lot. CW0103S) was purchased from Beijing ComWin Biotech Co., Ltd.

\subsection{Establishment and Screening of Model}

Sixty male Wistar rats $(280 \pm 20 \mathrm{~g})$ were provided for the experiments. Fifty rats were microinjection of composited $\mathrm{A} \beta$ on the right lateral cerebral ventricle and marked as composited $\mathrm{A} \beta$-treated rats. Ten rats were designed as sham operation. The rats were anaesthetized with isoflurane and fixed on brain stereotaxic apparatus. On the first $\mathrm{d}$ of the operation, as shown in $\mathrm{Wu}$ xiaoguang ${ }^{[8]}$ surgical methods, the bregma was the origin of coordinates. The first point [posterior $(\mathrm{P}): 2.0 \mathrm{~mm}$ to the bregma, lateral $(\mathrm{L}): 1.4 \mathrm{~mm}$ to the midline, and ventral $(\mathrm{V}): 4.6 \mathrm{~mm}$ to the skull] were microinjected into $10 \mathrm{ng}(1 \mathrm{ul})$ RHTGF- $\beta 1$. The second point [posterior (P): $0.8 \mathrm{~mm}$ to the bregma, lateral (L): $2.0 \mathrm{~mm}$ to the midline, and ventral $(\mathrm{V}): 4.6 \mathrm{~mm}$ to the skull] is for inserting the catheter, which apply to microinject with $4 \mu \mathrm{g}$ $(1 \mu \mathrm{L}) \mathrm{A} \beta_{25-35}$ for $14 \mathrm{ds}$ in the morning and $3 \mu \mathrm{L} \mathrm{AlCl}_{3}(1 \%)$ for $5 \mathrm{ds}$ in the afternoon, respectively on the second $\mathrm{d}$ of operation. The third point [Front $(\mathrm{F}): 2.0 \mathrm{~mm}$ to the bregma, lateral $(\mathrm{L}): 1.5 \mathrm{~mm}$ to the midline] is used to fixation. The sham operation group was received an equal volume of saline microinjection.

After $45 \mathrm{~d}$ of the operation, all rats were screened for memory impairment with Morris water maze. The training conduced twice a d for $4 \mathrm{~d}$, once near and once far, for $4 \mathrm{~d}$. The latency was the time for the rats to find the hidden platform. The learning score on the $\mathrm{d} 4$ was as the model screening index, the success rate of this experimental model is $81.25 \%$.

\subsection{Grouping, Administration and Sampling of Animals}

The successful model rats were divided into model group and three doses SSF groups randomly. Rats in the drug group intragastric administration SSF with 35,70 and $140 \mathrm{mg} / \mathrm{kg}$ for 30 consecutive ds. Rats 
in model group and the sham operation group were given an equal volume of saline. Rats were given SSF by intragastric administration for $31 \mathrm{~d}$, corresponded to $\mathrm{d} 76$ after the operation. All rats were decapitated $40 \mathrm{~min}$ after the last administration of SSF. The brain of the rats were placed on ice and the hippocampus and cerebral cortex were separated, which were wrapped with tinfoil, respectively, and stored at $-86^{\circ} \mathrm{C}$ for subsequent Western blotting detection.

\subsection{Western Blotting Detects P-tau (Thr205), P-tau (Thr212), P-tau (Ser202), P-tau (Ser205), P- tau (Ser214) and P-tau (Ser356) Proteins Expression Levels in Hippocampus and Cerebral Cortex of Rats}

The hippocampus and cortex tissues of rats were added to RIPA lysis buffer (10\% PMSF), homogenized, centrifuged, and the supernatant was taken. The concentration of the target protein was determined with BCA method. The total protein of hippocampal and cerebral cortex tissues of each group were extracted by RIPA lysis buffer (containing 10\% PMSF). Then, BCA method is used to determine sample protein concentration. The protein was transferred to a polyvinylidene difluoride (PVDF) membrane by wet transferred after SDS-PAGE electrophoresis 2 hours and observed the effect of membrane transfer with ponceau stain liquid, then sealed by $5 \%$ skimmed milk powder for 3 hours. The PVDF membrane was incubated in primary antibody of p-tau (Thr205), p-tau (Thr212), p-tau (Ser202), p-tau (Ser205), p-tau (Ser214) and p-tau (Ser356) at a certain proportion overnight at $4^{\circ} \mathrm{C}$. This PVDF membrane was washed with TBST for 3 times, 6 min of each, and then incubated with secondary antibody for $40 \mathrm{~min}$. Finally, this PVDF membrane was exposed and developed with chemiluminescence reagent ECL. Quantity One 4.6.2 software was used to analyze the protein bands after scanning the film. The gray value ratio of the target protein to the internal reference $\beta$-actin was regarded as the relative expression levels of the target protein.

\subsection{Statistical Analyses}

All data were analyzed using SPSS17.0 statistical software and performed as mean \pm SD. One-way analysis of variance (ANOVA) followed by the Duncan's multiple-range test was used to analyze group differences in the data from all the experiments, and $\mathrm{P}<0.05$ was considered statistically significant.

\section{Results}

\subsection{Effect of SSF on the Expression of P-tau (Thr205) Proteins in Hippocampus and Cerebral Cortex of Rats}

Figure 1 shows compared with the sham group, the protein expression of p-tau (Thr205) decreased by $12.7 \%$ and $111.02 \%(\mathrm{P}<0.01)$ in both the hippocampus and cerebral cortex, respectively. However, three doses of SSF can regulate the p-tau (Thr205) expression level in both hippocampus and cerebral cortex varying degrees. Compared with the model group, the protein expression of p-tau (Thr205) were decreased by $60.69 \%$ (35 mg/kg SSF, P < 0.01), 47.06\% (70 mg/kg SSF, P < 0.01), and $57.03 \%$ (140 $\mathrm{mg} / \mathrm{kg} \mathrm{SSF}, \mathrm{P}<0.01)$ in the cerebral cortex, respectively. For the hippocampus, the p-tau (Thr205) protein was also decreased but there was no significant difference with three doses of SSF treated.

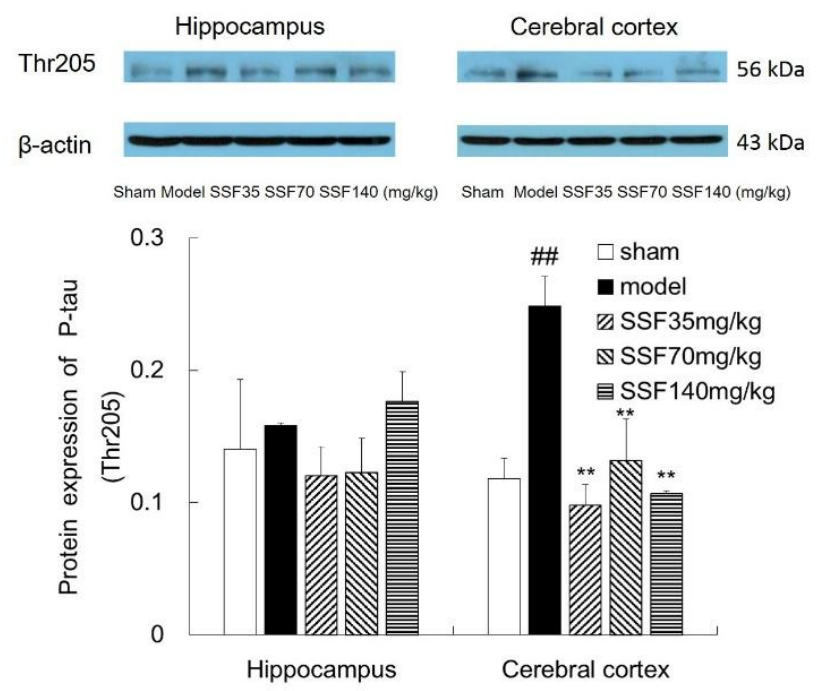

Figure1. The effect of SSF on the expression of p-tau (Thr205) proteins in hippocampus and cerebral cortex of rats with composited $A \beta$. Mean $\pm S D . n=3 .{ }^{* *} p<0.01$ vs model group. 


\subsection{Effect of SSF on the Expression of P-tau (Thr212) Proteins in Hippocampus and Cerebral Cortex of Rats}

As shown in Figure 2, the expression of p-tau (Thr212) was increased by $5.32 \%$ and $48.17 \%$ in both the hippocampus and cerebral cortex of the model group, as compared with the sham group, respectively. Compared with the model group, when the rats intracerebroventricularly injected with composited A $\beta$ were treated with 35,70 , and $140 \mathrm{mg} / \mathrm{kg}$ SSF for $30 \mathrm{ds}$. The expression of $\mathrm{p}$-tau (Thr212) was broadened $74.41 \%$ (hippocampus, $\mathrm{P}<0.01$ ) and $62.99 \%$ (cerebral cortex, $\mathrm{P}>0.05$ ) by treatment of $35 \mathrm{mg} / \mathrm{kg} \mathrm{SSF}$, respectively, increased $24.12 \%$ (hippocampus, $\mathrm{P}>0.05$ ) and $69.15 \%$ (cerebral cortex, $\mathrm{P}>0.05$ ) by treatment of $140 \mathrm{mg} / \mathrm{kg} \mathrm{SSF}$, respectively. The expression of $\mathrm{p}$-tau (Thr212) protein has no significant change with $70 \mathrm{mg} / \mathrm{kg}$ SSF treated.

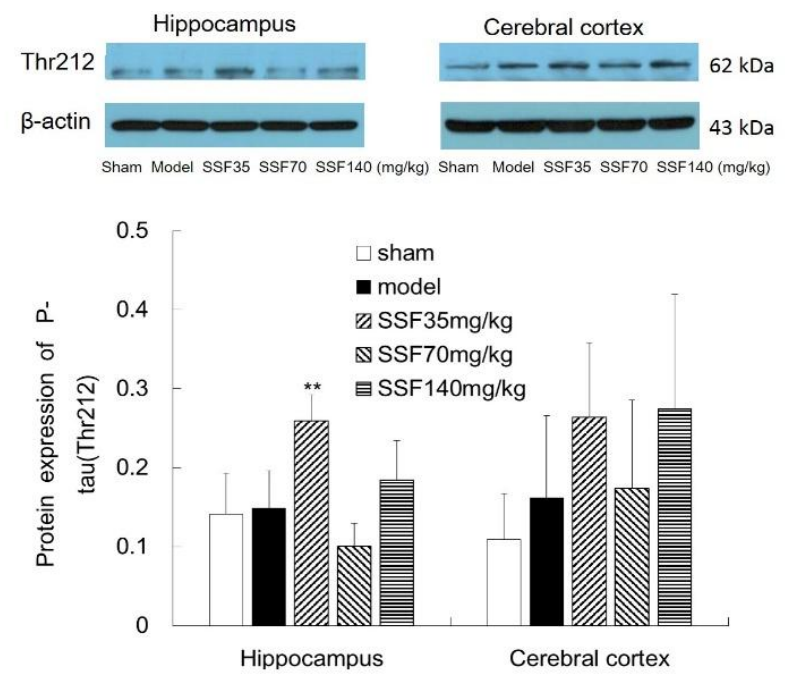

Figure2. The effect of SSF on the expression of p-tau (Thr212) proteins in hippocampus and cerebral cortex of rats with composited $A \beta$. Mean $\pm S D . n=3 .^{* *} p<0.01$ vs model group.

\subsection{Effect of SSF on the Expression of P-tau (Ser202) Proteins in Hippocampus and Cerebral Cortex of Rats}

Figure 3 shows the expression of p-tau (Ser202) proteins in hippocampus and cerebral cortex of rats. Compared with the sham group, the expression of p-tau (Ser202) was significantly reduced by $81.7 \%$ (hippocampus, $\mathrm{P}<0.01$ ) and $18.13 \%$ (cerebral cortex, $\mathrm{P}<0.05$ ) in the model group, respectively. Compared with the model group, three doses of SSF attenuated the expression of p-tau (Ser202) by $23.46 \%$ (35 mg/kg SSF, P < 0.05), 92.73\% (70 mg/kg SSF, P < 0.01), and 50.47\% (140 mg/kg SSF, P $<0.01)$ in the cerebral cortex, respectively. However, there was no significant difference in the expression of p-tau (Ser202) protein in the hippocampus.

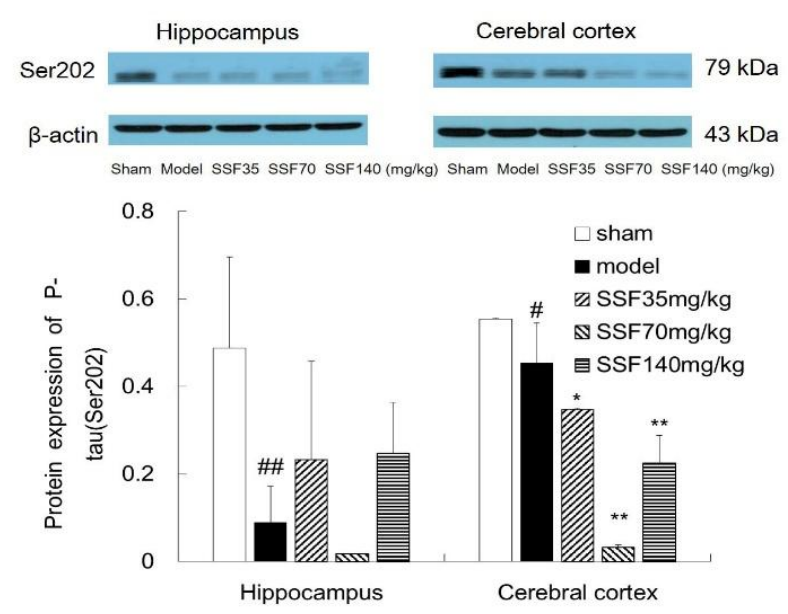

Figure3. The effect of SSF on the expression of p-tau (Ser202) proteins in hippocampus and cerebral cortex of rats with composited AB. Mean $\pm S D . n=3 .{ }^{\#} p<0.01$ vs sham group; ${ }^{*} p<0.05$ vs model group; ${ }^{* *} p<0.01$ vs model group. 


\subsection{Effect of SSF on the Expression of P-tau (Ser205) Proteins in Hippocampus and Cerebral Cortex of Rats}

The expression of p-tau (Ser205) proteins in hippocampus and cerebral cortex of rats as in Figure 4 showed. Compared with the sham group, intracerebroventricular injection of composited $A \beta$ can reduce the p-tau (Ser205) values by $18.13 \%(\mathrm{P}<0.01)$ in the cerebral cortex of the model group. While, there was no markedly changed in the hippocampus. Compared with the model group, three doses of SSF decreased the expression of p-tau (Ser205) proteins by $40.32 \%$ (35 mg/kg SSF, P < 0.01), $31.16 \%$ (70 $\mathrm{mg} / \mathrm{kg} \mathrm{SSF}, \mathrm{P}<0.01)$, and $35.70 \%$ (140 mg/kg SSF, P < 0.01) in the cerebral cortex, respectively. 140 $\mathrm{mg} / \mathrm{kg}$ SSF reduced p-tau (Ser205) protein expression by $19.13 \%(\mathrm{P}<0.01)$, but the expression of $\mathrm{p}$ tau (Thr212) has no significant change with 35 and $70 \mathrm{mg} / \mathrm{kg}$ SSF treated in hippocampus.

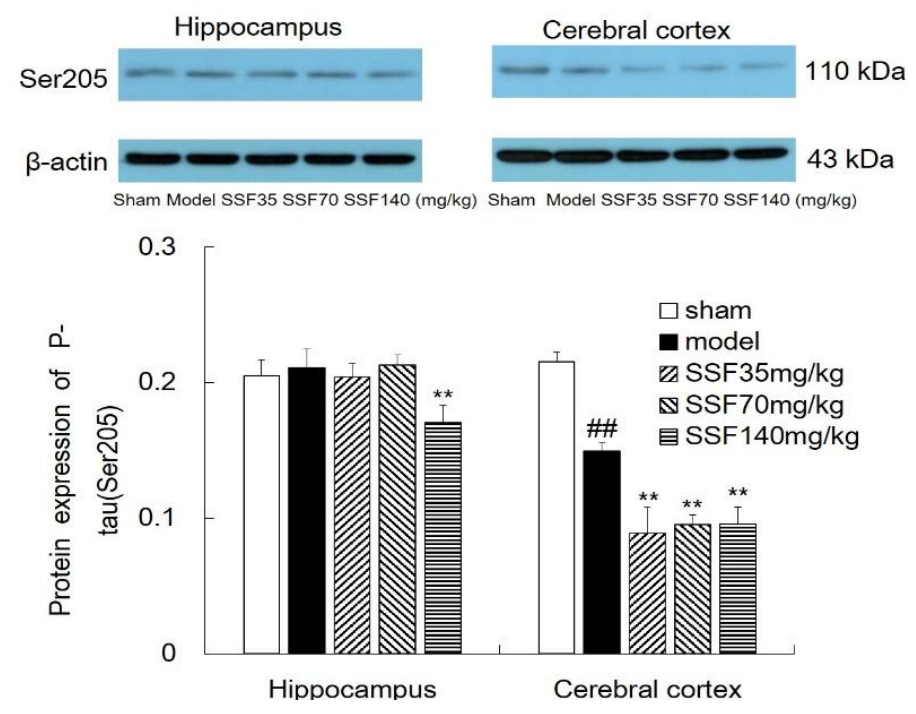

Figure4. The effect of SSF on the expression of p-tau (Ser205) proteins in hippocampus and cerebral cortex of rats with composited AB. Mean $\pm S D . n=3 .{ }^{\# p} p<0.01$ vs sham group; ${ }^{* *} p<0.01$ vs model group.

3.5 Effect of SSF on the Expression of P-tau (Ser214) Proteins in Hippocampus and Cerebral Cortex of Rats

Figure 5 shows that intracerebroventricular injection of composited $A \beta$ can increase p-tau (Ser214) protein expression by $30.13 \%(\mathrm{P}<0.01)$ and $1.95 \%$ in both the hippocampus and the cerebral cortex. However, three doses of SSF can regulate the p-tau (Ser214) expression level in both hippocampus and cerebral cortex varying degrees. Compared with the model group, 70 and $140 \mathrm{mg} / \mathrm{kg} \mathrm{SSF}$ decreased the protein expression of p-tau (Ser214) by $16.58 \%(\mathrm{P}<0.01)$ and $22.21 \%(\mathrm{P}<0.01)$ in the hippocampus, respectively. 35 and $140 \mathrm{mg} / \mathrm{kg}$ SSF attenuated the p-tau (Ser214) protein expression by $21.37 \%$ (P < $0.01)$ and $27.41 \%(\mathrm{P}<0.01)$, respectively in the cerebral cortex. In addition, p-tau (Ser214) protein expression has no significant difference by $35 \mathrm{mg} / \mathrm{kg} \mathrm{SSF}$ in the hippocampus and $70 \mathrm{mg} / \mathrm{kg} \mathrm{SSF}$ in the cerebral cortex.

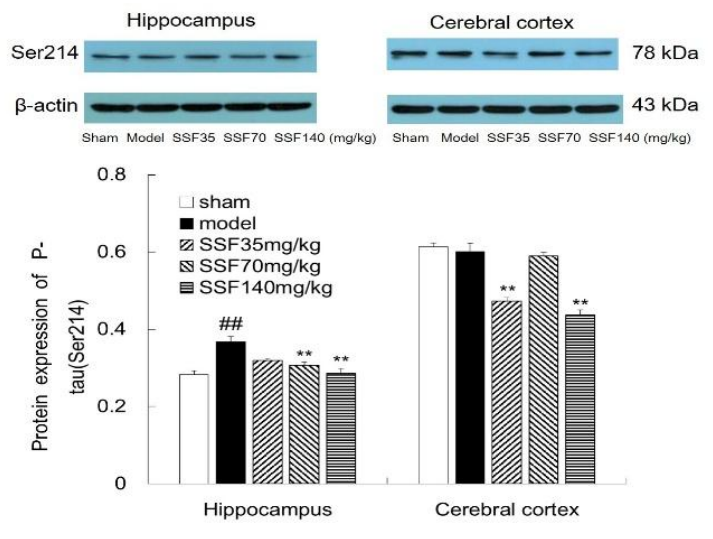

Figure5. The effect of SSF on the expression of p-tau (Ser214) proteins in hippocampus and cerebral cortex of rats with composited $A \beta$. Mean $\pm S D . n=3 .{ }^{\# p} p<0.01$ vs sham group; ${ }^{* *} p<0.01$ vs model group. 


\subsection{Effect of SSF on the Expression of P-tau (Ser356) Proteins in Hippocampus and Cerebral Cortex of Rats}

Figure 6 shows the expression of p-tau (Ser356) proteins in hippocampus and cerebral cortex of rats. Compared with the sham group, intracerebroventricular injection of composited $A \beta$ can decrease $p$-tau (Ser356) protein expression by $26.05 \%(\mathrm{P}<0.01)$ in the cerebral cortex. While, there was no significant change in the hippocampus. Compared with the model group, the expression of $\mathrm{p}$-tau (Ser356) protein was decreased by $57.03 \%(\mathrm{P}<0.01)$ and $41.79 \%(\mathrm{P}<0.01)$ by treatment of 35 and $70 \mathrm{mg} / \mathrm{kg} \mathrm{SSF}$, and increased by $15.92 \%(\mathrm{P}<0.01)$ by $140 \mathrm{mg} / \mathrm{kg}$ SSF treated in the cerebral cortex. For the hippocampus, the expression of p-tau (Ser356) was reduced by $44.34 \%$ (P< 0.01 ) by $70 \mathrm{mg} / \mathrm{kg}$ SSF treated, 35 and $140 \mathrm{mg} / \mathrm{kg} \mathrm{SSF}$ can decrease p-tau (Ser356) protein expression but no significant different.

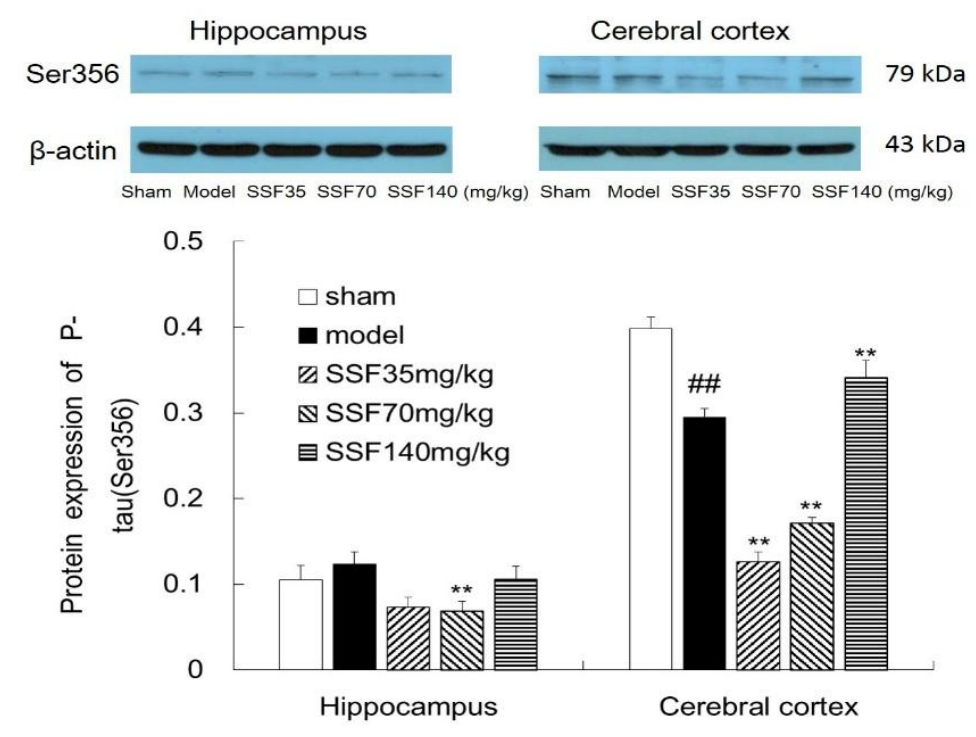

Figure6. The effect of SSF on the expression of p-tau (Ser356) proteins in hippocampus and cerebral cortex of rats with composited AB. Mean $\pm S D . n=3 .{ }^{\# p} p<0.01$ vs sham group; ${ }^{* *} p<0.01$ vs model group.

\section{DISCUSSION}

The pathogenesis of $\mathrm{AD}$ is the result of many pathogenic factors. Its main pathological feature includes extracellular senile plaque (SP) and intracellular NFTs. The former is composed of aggregated A $\beta$, and the latter is composed of aggregates of hyperphosphorylated tau protein. $A \beta$ deposition is the basis for the formation of AD. However, cognitive impairment was found in $\mathrm{A} \beta$ precursor transgenic mice, which must be accompanied by abnormal changes in tau protein phosphorylation ${ }^{[9]}$. Tau protein is a microtubule-related protein in the nervous system. Normally, tau protein is soluble, mainly exists in the axons of neurons. It can combine with tubulin to promote the formation stability of microtubules, participate in regulating the material transport of nerve cell axons. Under pathological conditions, tau protein is phosphorylated at multiple sites to make it from soluble to insoluble, and then leading to NFTs, which can induce the nerve cells structure damaged and dysfunction and finally result in degenerative disease ${ }^{[10,11]}$. At present, there are about 40 abnormal phosphorylation sites in tau protein that have been identified, including Thr181, Ser199, Ser202, Thr205, Thr212, Ser205, Thr214, Ser235, Ser356, Ser396, Ser404 and others. The experimental studies in vivo and in vitro have found that phosphorylated tau protein can disseminate among cells, transmit between neurons through synapses $[12,13]$, and eventually spread throughout the brain.

CDK-5 is a protein kinase that only plays a part in the nervous system. It can be promots the phosphorylation of Tau protein at multiple Ser/Thr sites including Thr205, Thr212, Ser202, Ser205, Ser214, Ser356 and others ${ }^{[14-17]}$. Phosphorylation at Ser205 was correlated with age ${ }^{[18]}$. The decrease of phosphorylation level of Ser205 with composited $A \beta$ in this study may be related to the age of rats. Both AT8 (Ser202/Thr205 dual phosphorylation sites) and AT100 (Thr212/Ser214 dual phosphorylation sites) are the tau specific monoclonal antibodies, which is recognized as abnormal phosphorylated tau proteins and specific markers of $\mathrm{AD}{ }^{[19-20]}$. In the present study, the levels of phosphorylation at Thr205 and phosphorylation at Ser202 in rats induced by composited A $\beta$ were 
increased, which indicated abnormal phosphorylation tau protein. Phosphorylation at Ser214 of tau protein has a protective effect on the aggregation of abnormal tau protein. Phosphorylation at Ser214 alone can effectively reduce tau's affinity for microtubule. Besides, phosphorylation at Thr212 is difficult to produce solely ${ }^{[21,22]}$. Phosphorylation at Ser214 protects Thr212 from phosphorylation, and AT100 epitopes do not form. Moreover, experts predict that phosphorylation of Thr212 can promote phosphorylation of Ser214 ${ }^{[23]}$. In the present study, intracerebroventricular injection of composited A $\beta$ can increase Tau protein phosphorylation at Ser214, but phosphorylation at Thr212. It suggests that composited $\mathrm{A} \beta$ decreased affinity of tau protein to microtubules, and the phosphorylation of AT100 does not perform. Ser356 of tau protein is located in the microtubule-binding region. The interaction between phosphorylated tau and microtubules is significantly reduced ${ }^{[24]}$. Phosphorylation at Ser356 of Tau can block the interaction between tau protein and $A \beta^{[25]}$. Tau hyperphosphorylation is not only related to the activity of tau protein and related kinases, but also plays an important role in the posttranslational modification of glycosylation, acetylation, oxidation and ubiquitination for tau. In addition, intracerebroventricular injection of composited $A \beta$ can reduce the affinity of tau protein to microtubules and accelerate tau phosphorylation transmission.

The present results showed that intracerebroventricular injection of composited $\mathrm{A} \beta$ can hyperphosphorylate at Thr205 and Ser214 sites of tau protein in the rats' brain. SSF can modulate tau phosphorylation, especially inhibit the tau phosphorylation at Ser205 and Ser214 sites. Together with our previous researches ${ }^{[26,27]}$, the present study provided an evidence that the improvements of SSF in neuroprotection and memory deficits may be related to its regulation in multiple sites hyperphosphorylation of tau, especially inhibition at Thr205 and Ser214 sites of tau protein.

\section{AUTHORS CONTRIBUTION}

Shang Yazhen conceived and designed the experiment. Liu Qianqian, Ding Shengkai, and Zhang Hui conducted the test and wrote the manuscript.

\section{ACKNOWLEDGEMENTS}

The authors wish to thank Hebei Provincial Natural Science Foundation (No. C2009001007, H2014406048, H2019406063), Hebei Provincial Administration of Traditional Chinese Medicine (No. 05027, 2014062), Hebei Provincial Education Department (No. ZD20131022, 2019057), Hundred Outstanding Innovated Talents, Hebei Province (First Batch), Key Subject Construction Project of Hebei Provincial College, (J. J. G. No. [2013]4), the Key Development Subject of Pharmacology of Traditional Chinese Medicine of Chengde Medical College (No. [2020]49) and Science and Technology Innovation Team Construction Project of Chengde Medical College, China. (No. [2020]50) for financial support.

\section{REFERENCES}

[1] Wang JC, Liu HY, Cao YP. Tau protein and Alzheimer's disease. Chinese Journal of Tissue Engineering Research, 2020, 24(17):2775-2781.

[2] Teresa, Gómez-Isla, Richard, et al. Neuronal loss correlates with but exceeds neurofibrillary tangles in Alzheimer's disease. Annals of Neurology, 2004, 41(1):17-24.

[3] Goedert M. Tau filaments in neurodegenerative diseases. FEBS Letters, 2018, 592(14):2383-2391.

[4] Chandra A, Polytimi-Eleni Valkimadi, Pagano G, et al. Applications of amyloid, tau, and neuroinflammation PET imaging to Alzheimer's disease and mild cognitive impairment. Human Brain Mapping, 2019, 40(18):54245442 .

[5] Zhang SF, Dong YC, Zhang XF. Flavonoids from Scutellaria attenuate okadaic acid-induced neuronal damage in rats. Brain Injury, 2015, 29(11):1379-1382.

[6] Wang XQ, Gao Y, Dong YC, et al. Flavonoids from stem and leaf of Georgi inhibit PHF abnormality and regulatory mechanism of protein phosphatase in rats' brain induced by okadaic acid. Chinese Journal of Pathophysiology, 2018, 34(1):94-100.

[7] Gao Y, Wang XQ, Ma S, et al. Flavonoids from Stem and Leaf of Scutellaria Baicalonsis Georgi Inhibit the Phosphorylation on Multi-sites of Tau Protein Induced by Okadaic Acid and the Regulative Mechanism of Protein Kinases in Rats. Combinatorial Chemistry \& High Throughput Screening, 2020.

[8] Wang XQ, Gao Y, Dong YC, et al. Flavonoids from stem and leaf of Scutellaria baicalonsis Georgi inhibit PHF abnormality and regulatory mechanism of protein phosphatase in rats' brain induced by okadaic acid. Chinese Journal of Pathophysiology, 2018, 34(001):94-100. 
[9] Amniai L, Barbier P, Sillen A, et al. Alzheimer disease specific phosphoepitopes of Tau interfere with assembly of tubulin but not binding to microtubules. Faseb Journal. 2009, 23(4):1146-1152.

[10]Wang Y, Mandelkow E. Tau in physiology and pathology. Nature Reviews Neuroscience, 2016, 17(03):2235 .

[11] Han DY, Yang N, Guo XY. The role of microglia activation by pathological Tau protein in cognitive impairment. International Journal of Anesthesiology and Resuscitation, 2020, 41(002):221-224.

[12] Takeda S, Wegmann S, Cho H, et al. Neuronal uptake and propagation of a rare phosphorylated highmolecular-weight tau derived from Alzheimer's disease brain. Nature Communications, 2015, 6:8490.

[13] Brunello CA, Merezhko M, Uronen RL, et al. Mechanisms of secretion and spreading of pathological tau protein. Cellular and Molecular Life ences, 2020, 77(9):1721-1744.

[14] Lopes JP, Agostinho P. Cdk5: multitasking between physiological and pathological conditions. Progress Neurobiology, 2011, 94(1):49-63.

[15] Wang JZ, Xia YY, Grundke-Iqbal I, et al. Abnormal hyperphosphorylation of tau: sites, regulation, and molecular mechanism of neurofibrillary degeneration. Journal of Alzheimer's disease, 2013, 33:123-139.

[16] Lund ET, Mckenna R, Evans DB, et al. Characterization of the in vitro phosphorylation of human tau by tau protein kinase II (cdk5/p20) using mass spectrometry. Journal of Neurochemistry, 2001, 76(4):1221-1232.

[17] Chen YQ, Tian XH, Jia BQ, et al. The mechanism of abnormal phosphorylation of tau protein in Alzheimer's disease. Journal of Brain and Nervous Diseases, 2019, 27(10):53-58.

[18] Bodea LG, Evans HT, Ann VJ, et al. Accelerated aging exacerbates a pre-existing pathology in a tau transgenic mouse model. Aging Cell, 2017, 16(2):377-386.

[19] Thomas JM, Alexey T, Robin E, et al. Epitope mapping and structural basis for the recognition of phosphorylated tau by the anti-tau antibody AT8. Proteins: Structure, Function, and Genetics, 2016, 84(4):42734.

[20] Gil L, Federico C, Pinedo F, et al. Aging dependent effect of nuclear tau. Brain Research, 2017, 1677:129137.

[21] Ksiezak-Reding H , Pyo HK, Feinstein B, et al. Akt/PKB kinase phosphorylates separately Thr212 and Ser214 of tau protein in vitro. Biochim Biophys Acta, 2003, 1639(3):159-168.

[22] Schneider A, Biernat J, Von Bergen M, et al. Phosphorylation that detaches tau protein from microtubules (Ser262, Ser214) also protects it against aggregation into Alzheimer paired helical filaments. Biochemistry, 1999, 38(12):3549-3558.

[23] Qingyi Zheng-Fischhöfer, Biernat J, Mandelkow EM, et al. Sequential phosphorylation of Tau by glycogen synthase kinase-3beta and protein kinase A at Thr212 and Ser214 generates the Alzheimer-specific epitope of antibody AT100 and requires a paired-helical-filament-like conformation. European Journal of Biochemistry, 2010, 252(3):542-552.

[24] Ando K, Oka M, Ohtake Y, et al. Tau phosphorylation at Alzheimer's disease-related Ser356 contributes to tau stabilization when PAR-1/MARK activity is elevated. Biochemical \& Biophysical Researchunications, 2016, 478(2):929-934.

[25] Derreumaux P, Man VH, Wang J, et al. Tau R3-R4 Domain Dimer of the Wild Type and Phosphorylated Ser356 Sequences. I. In Solution by Atomistic Simulations. The Journal of Physical Chemistry B, 2020, 124(15):2975-2983.

[26] Chen JJ, Zhao HX, Guo K, et al. The regulation mechanism of Scutellaria Baicalensis Georgi stem and leaf flavonoids in alleviating memory impairment induced by compound A and inhibiting neuronal apoptosis in rats. Chinese Journal of New Drugs 2016, 25(22):2627-2636.

[27] Zhang Hui, Ding Shengkai, Liu Qianqian, et al. $\mathrm{Ca}^{2+}-$ Camk-CREB Mediates the Effect of Flavonoids from Scutellaria Baicalensis Stems and Leaves on the Decrease of Neuroregeneration Induced by Composited A $\beta$ in Rats. International Journal of Medicinal Plants and Natural Products. 2019, 5(4):18-28.

Citation: Shang Yazhen, et.al., (2020). "Effect of Scutellaria Baicalensis Georgi Stems and Leaves Flavonoids on Multisite Phosphorylation of tau Protein Induced by Composited A $\beta$ in Rats". International Journal of Medicinal Plants and Natural Products (IJMPNP), 6(4), pp.1-8. https://doi.org/ 10.20431 /24547999.0604001

Copyright: (C) 2020 Authors. This is an open-access article distributed under the terms of the Creative Commons Attribution License, which permits unrestricted use, distribution, and reproduction in any medium, provided the original author and source are credited. 